\title{
BMJ Open Realist evaluation of Autism ServiCe Delivery (RE-ASCeD): which diagnostic pathways work best, for whom and in what context? Findings from a rapid realist review
}

\author{
Vanessa Abrahamson (D) , ${ }^{1}$ Wenjing Zhang (D) , ${ }^{1}$ Patricia M Wilson (D) , ${ }^{1}$ \\ William Farr (D) , ${ }^{2,3}$ Venkat Reddy (D) , Jeremy Parr (D) , ${ }^{5,6,7}$ Anna Peckham (D) , \\ Ian Male (1) 2,3
}

To cite: Abrahamson V, Zhang W, Wilson PM, et al. Realist evaluation of Autism ServiCe Delivery (RE-ASCeD): which diagnostic pathways work best, for whom and in what context? Findings from a rapid realist review. BMJ Open 2021;11:e051241. doi:10.1136/ bmjopen-2021-051241

- Prepublication history and additional supplemental material for this paper are available online. To view these files, please visit the journal online (http://dx.doi.org/10.1136/ bmjopen-2021-051241).

Received 15 March 2021 Accepted 20 0ctober 2021

Check for updates

(c) Author(s) (or their employer(s)) 2021. Re-use permitted under CC BY-NC. No commercial re-use. See rights and permissions. Published by BMJ.

For numbered affiliations see end of article.

Correspondence to Dr Vanessa Abrahamson; v.j.abrahamson@kent.ac.uk

\section{ABSTRACT}

Objectives Waiting times in the UK for an autism diagnostic assessment have increased rapidly in the last 5 years. This review explored research (including 'grey' literature) to uncover the current evidence base about autism diagnostic pathways and what works best, for whom and in what circumstances, to deliver high quality and timely diagnosis. Design We performed a Rapid Realist Review consistent with recognised standards for realist syntheses. We collected 129 grey literature and policy/guidelines and 220 articles from seven databases (January 2011-December 2019). We developed programme theories of how, why and in what contexts an intervention worked, based on cross comparison and synthesis of evidence. The focus was on identifying factors that contributed to a clearly defined intervention (the diagnostic pathway), associated with specific outcomes (high quality and timely), within specific parameters (Autism diagnostic services in Paediatric and Child \& Adolescent Mental Health services in the UK). Our Expert Stakeholder Group, including representatives from local parent forums, national advocacy groups and clinicians, was integral to the process.

Results Based on 45 relevant articles, we identified 7 programme theories that were integral to the process of diagnostic service delivery. Four were related to the clinical pathway: initial recognition of possible autism; referral and triaging; diagnostic model; and providing feedback to parents. Three programme theories were pertinent to all stages of the referral and diagnostic process: working in partnership with families; interagency working; and training, service evaluation and development.

Conclusions This theory informed review of childhood autism diagnostic pathways identified important aspects that may contribute to efficient, high quality and familyfriendly service delivery. The programme theories will be further tested through a national survey of current practice and in-depth longitudinal case studies of exemplar services. Trial registration number NCT04422483.

\section{INTRODUCTION}

The number of children and young people (CYP) diagnosed with autism spectrum
Strengths and limitations of this study

- This realist review focused on reviewing and synthesising recent evidence to determine what approaches to autism diagnostic assessment worked best, for whom and in what context. The approach is better suited than more empirical methods that assume there is one model to suit all situations.

- Our Expert Stakeholder Group and parent representatives engaged with all stages of the review and enabled an iterative approach to identifying relevant literature and refining our findings.

- As appropriate to our research question, we limited the search to UK literature but may have missed relevant literature from similar health systems. Although synthesis was based on UK literature, we have considered how this relates to relevant international literature.

disorder (autism) has increased significantly in recent years ${ }^{1-3}$ with a median age for diagnosis of 55 months. ${ }^{4}$ This international phenomena is reflected in increasing pressures on diagnostic assessment and long waiting times in some services, ${ }^{5}$ with associated family dissatisfaction. ${ }^{6}$ The UK National Health Service (NHS) Long Term Plan ${ }^{7}$ highlighted the need for research to identify the most effective ways to improve timely access to diagnosis while maintaining high-quality assessment for this service user group.

Autism is characterised by persistent severe deficits in social interaction, social communication, and restricted, repetitive, inflexible patterns of behaviour and interests, ${ }^{8}$ although the level of symptoms varies considerably between individuals. It is commonly associated with other neurodevelopmental and mental health conditions, such as anxiety, Attention 
Deficit Hyperactivity Disorder (ADHD) and developmental language disorder, ${ }^{9-11}$ making reliable diagnosis a complex process. National guidelines for Autism in the $\mathrm{UK}^{12}$ recommend multidisciplinary assessment, with the skills to consider both the presence of other neurodevelopmental and mental health conditions (eg, ADHD, anxiety disorders), and coexisting conditions (eg, eating or sleeping related). However, this holistic assessment is time consuming and costly. ${ }^{134}$ There are significant variations between diagnostic pathways, which some have defined as 'complex interventions for mutual decision making, organisation and standardization of predictable care for a well-defined group of patients during a welldefined period', ${ }^{15}$ and only limited evidence of which pathways work best, for whom and in what circumstances.

Although the formal research base is limited, some local providers have already reconfigured their services to address these issues. ${ }^{16-18}$ However, robust evidence is needed to identify which care pathways, in which contexts, have the potential to meet the growing demand for diagnostic assessment in a timely, clinically valid and familyfriendly way. This Rapid Realist Review (RRR), the first step in a national Realist Evaluation of Autism ServiCe Delivery (RE-ASCeD), aimed to explore how particular approaches aspired to deliver high quality and timely autism diagnostic services. ${ }^{19}$ High quality was defined as compliant with National Institute for Health and Care Excellence (NICE) guidelines. ${ }^{12}$ 'Timely' refers to diagnostic pathways that must be started within 3 months of referral, in-line with NICE guidelines, ${ }^{1}$ and last no more than one calendar year.

This study aimed to explore research evidence about autism diagnostic pathways to determine what works best, for whom and in what circumstances. The RRR aimed to use the literature to address the following questions:

1. How do various pathways of autism diagnostic and support services address the differing needs of service users and what contexts and mechanisms affect their ability to do so?

2. How do different pathways of autism diagnostic and support services improve service user diagnostic experience?

3. What aspects of implementation, staffing and organisational context influence how care pathways for autism diagnostic and support services operate?

\section{METHOD}

Autism diagnostic care pathways vary in terms of complex differences in local service configurations and settings, lending itself to realist review that can tease out contextual factors, resources and responses of those delivering and accessing the services. A systematic review may not be best matched to the heterogeneity of autism diagnostic services nor to capturing what is most helpful for policy decisions. Our focus was exploring solutions, so we did not focus on wider constraints, already widely documented, and incorporating chronic underfunding; increasing caseloads; reduced training budgets; and recruitment/retention issues, particularly paediatricians, child psychiatrists, clinical psychologists and speech and language therapies (SALTs). ${ }^{20}{ }^{21}$ Similarly, we did not focus on causes of service user dissatisfaction, rather ways of addressing it.

A RRR is a well-established approach to synthesising evidence within a compressed time period and the key steps are consistent with the Realist And Meta-narrative Evidence Syntheses: Evolving Standards (RAMESES) standards for realist syntheses ${ }^{22}$; thus the difference is the timeframe, not the level of rigour. Additionally, RRR is explicitly designed to engage with stakeholders to accelerate the search process and validate findings. ${ }^{17}$ Our Expert Stakeholder Group included clinicians (consultant paediatricians, child psychology, SALT), policymakers and third sector advocacy groups (Council for Disabled Children and Autistica) who were involved in all stages of the process. ${ }^{19}$ Ethical approval was not required because stakeholders were acting as research advisers, not participants. ${ }^{23}$

Realist reviews do not seek to compare interventions, rather they present evidence as programme theories (PTs) which are key features of the service and describe what appears to lead to certain outcomes, ${ }^{24}$ often phrased as 'If... Then...' statements. PTs are supported by details of the context $(\mathrm{C})$, mechanisms $(\mathrm{M})$ and outcomes $(\mathrm{O})$. These relationships are presented as CMO configurations. ${ }^{25}$ A realist approach requires starting with an initial PT of what should work and what outcomes are expected from a complex intervention; our PT was based on NICE 2011 guidance, ${ }^{12}$ the project team and Expert Stakeholder Group:

If there is a MDT assessment by a team with competencies in child neurodevelopment and mental health (context), then Autism will be recognised as a complex condition that relies on detailed history and observation across settings (mechanism) to diagnose it. This will lead to accurate diagnosis, recognition of associated co-occurring conditions such as ADHD and intellectual disability (outcome), and the ruling out of complex differential diagnoses. This will also create, whilst not an explicit part of this project, an accurate picture of a child's strengths and needs to inform individualised packages of support and intervention through health, education and social care (outcome).

We worked backwards from the intended outcomes although we know in practice that complex interventions operating in different health and social care environments do not lead to the same outcomes across services because of differing contexts (eg, differences between services, ways of operationalising and differences in recipient populations). Therefore, what is required is an understanding of what needs to be in place (circumstances or context), to trigger mechanisms (that can be responses or 
resources) that lead to the desired (intended) outcomes or other unintended outcomes.

\section{Changes to protocol}

No changes to the review process proposed in the published protocol (https://bmjopen.bmj.com/ content/10/7/e037846).

\section{Search methods}

This RRR was carried out from 1 September 2019 to 30 June 2020 following RAMESES standards ${ }^{24}$ for realist reviews. Through discussions within the RE-ASCeD project team and with our expert stakeholders, we confirmed and refined the research questions and scope; prioritised areas for investigation; identified search terms; and collected grey literature, policy and guideline papers iteratively throughout the review.

Search terms were identified and developed with support from the RE-ASCeD project team and expert stakeholders. The primary search was conducted across Medline (Ovid), Embase (Ovid), PsycINFO (Ovid), Social Policy \& Practice (Ovid), CINAHL Plus (EBSCO), Cochrane Library and Web of Science (Clarivate) limited by date (2011-2019), language (English) and country (UK only). Our focus was a clearly defined intervention (the diagnostic pathway, from receipt of referral to diagnosis), associated with specific outcomes (high quality and timely) within a particular set of parameters (autism/ Child \& Adolescent Mental Health Services (CAMHS) in the UK). All study types were included. The search strategy was created by an information specialist (AP) using a combination of free text and MeSH index terms after iterative pilots in Medline and adapted for each database. Search strings were based on a combination of terms covering "Children", AND "Autism" AND how they "Relate to diagnostic pathway OR assessment". For full search terms, see online supplemental document 1 . Box 1 provides our inclusion/exclusion criteria.

Secondary searching was conducted iteratively throughout the review with input from our expert stakeholders. Two reviewers used papers identified in the primary and background search to look through reference lists for relevant articles; check forward citations; and search key authors and research teams to identify further literature, using Google scholar. Primary and background searches were restricted to UK only, given UK NHS context. On the advice of our expert stakeholders, we then reviewed high level national policy documents and guidelines and a few research articles from similar countries (USA, Canada, Australia, New Zealand) to help elucidate findings.

\section{Article selection and appraisal}

As shown in figure 1, we collected 294 articles from the primary search, 129 grey literature records suggested by the RE-ASCeD project team members and our expert stakeholders, with overall 338 items once duplicates removed. Furthermore, nine papers were collected via

\section{Box 1 Inclusion/exclusion criteria}

Inclusion criteria

- Children (preschool, primary or secondary school and adolescents) with Autism Spectrum Disorder (ASD) or Autism spectrum condition.

- UK healthcare system (England, Scotland, Wales and N. Ireland).

- Published 2011 onwards when the National Institute for Health and Care Excellence guidelines for recognition, referral and diagnosis of autism in under 19s (2011) was published.

- Relates to diagnostic pathway and model of service provision or relates to assessment process, for example, single discipline (paediatric consultant) or multidisciplinary.

Primary exclusion criteria:

- Non-UK based literature.

- Relates only to adult diagnostic pathway.

- Relates only to tertiary services.

- Only relates to treatment.

- Relates to support services only after diagnosis.

Secondary exclusion criteria:

- Descriptive or irrelevant commentary on materials we already included; no added insights relevant to context or mechanisms.

- Specific tools in terms of assessment tools or psychometric properties, for example, reliability/validity of the tool.

- Prevalence only studies.

- Studies only related to symptoms or aetiology.

- Articles about special needs in general, no mention of ASD (or ADHD).

- Duplicate material of Co-Investigators' (Co-Is) previous research, excluded by Co-Is.

- Conference paper with only abstract available.

- The data collected or published online before 2011.

iterative secondary searches by searching all publications for key authors using Google Scholar and consulting our Expert Stakeholder Group. Two researchers (VA and WZ) carried out screening in two stages: an initial stage by title and abstract and second stage by full text. Title sifting of papers that deemed 'relevant' or 'maybe relevant' from both stages was also cross checked by three team members (PW, WF and IM). Data extraction and appraisal were carried out by two researchers (VA and WZ) using a hybrid approach ${ }^{26}{ }^{27}$ : basic details from each included article $(n=79)$ were recorded; appraisal of evidence was based on concepts of relevance, rigour and richness,${ }^{2627}$ with highly relevant articles $(n=45$, including 9 from iterative secondary search) coded in NVivo. For $20 \%$ of papers, a series of calibration exercises were undertaken by the RRR Lead (PW). When two reviewers were uncertain about the extraction or appraisal of a paper, this was discussed with the RRR Lead. The quality and relevance of the selected papers were also assessed during the synthesis process by members from the RE-ASCeD project team.

Mapping the sources to test and develop PTs, we divided papers involved in NVivo analysis into three categories: (1) key papers that described a model of service delivery (eg, integrated neuro-developmental service) in detail and were conceptually rich, (2) 'medium' papers that mentioned a model with some useful information 


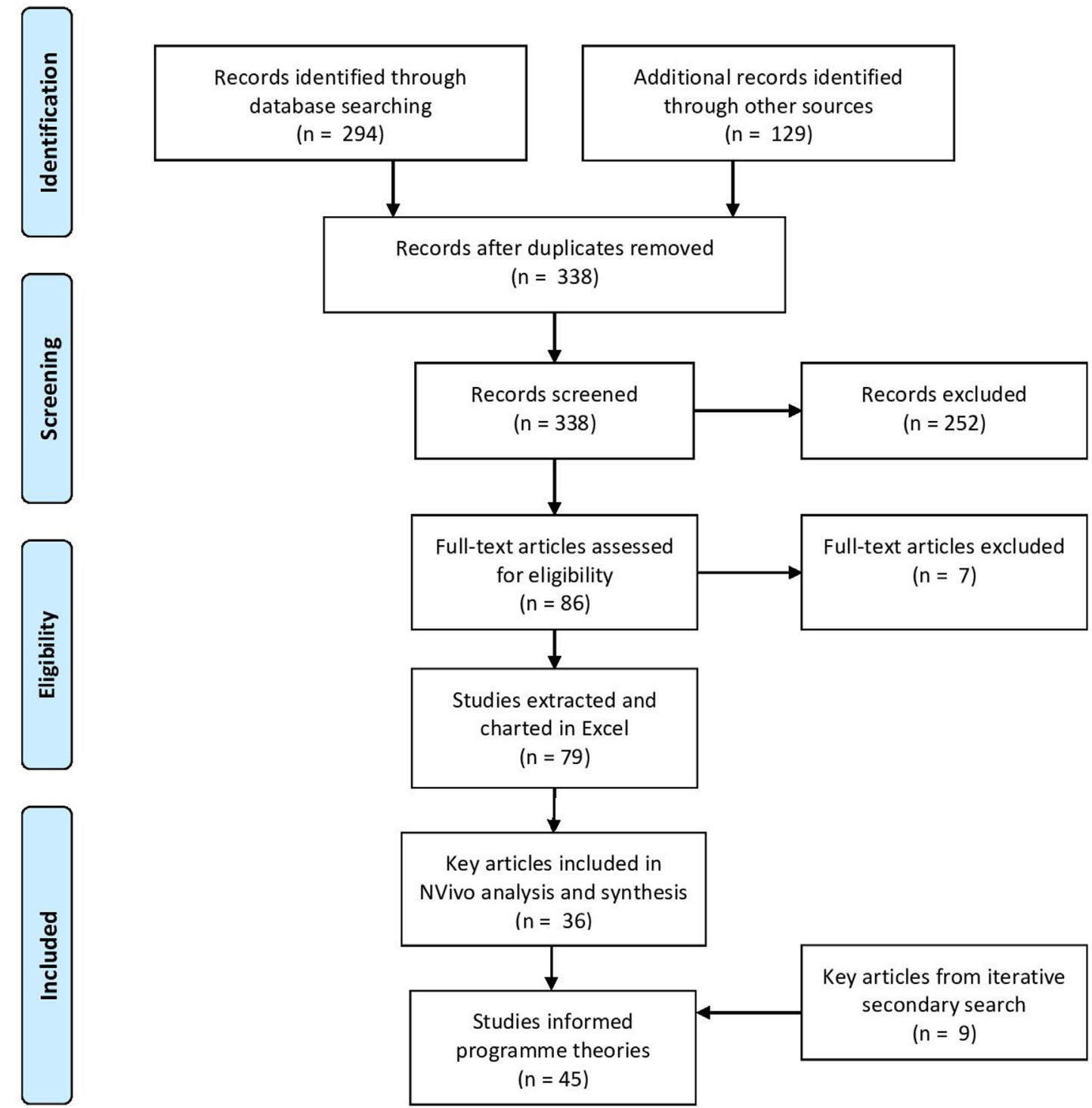

Figure 1 Search and review flow diagram.

but were not conceptually rich, (3) papers with a few 'nuggets' ${ }^{28}$ relevant to PTs. This helped us focus on key and medium papers (online supplemental document 2) that could contribute most to developing a conceptual framework $^{29}$ and refining PTs.

\section{Synthesis and refinement}

Based on analysis of individual papers, we then conducted cross-evidence comparisons to build PTs and confirm/ refute and refine CMO configurations; both synthesis and refining the evidence involved substantial discussion of 'contradictory' evidence, or unintended outcomes. We also consulted with our expert stakeholders iteratively during the review process and at a data interpretation workshop in April 2020. Our expert stakeholders collectively reviewed the PTs, provided feedback and were invited to identify any omissions based on their clinical experience. We also asked them to suggest any further literature to help elucidate PTs. Based on feedback collected from the data interpretation workshop, two reviewers (VA and WZ) checked and added new papers suggested by our expert stakeholders; refined the PTs and conceptual framework.

\section{Patient and public involvement}

Our Co-Investigators included a patient and public involvement (PPI) representative from a local parent organisation (West Sussex Parent Carer Forum) who was able to consult a wider group of families with lived experience and a parent who had previously managed Sussex Autism Support. Our PPI representatives were equal partners within the Expert Stakeholder Group. This helped focus the review on the questions they were most interested in answering and enabled the identification of salient grey or unpublished documents for review. ${ }^{30}$ PPI was embedded into the review protocol and was particularly helpful when synthesising and interpreting the data. A separate PPI Reference Group (all parents of CYP with autism), whose inception was delayed due to COVID-19, is integral to the wider project. 


\section{RESULTS}

We developed seven PTs, based on cross-comparison and synthesis of 45 highly relevant articles: the first four focused on referral and diagnostic process and the last three on cross-cutting themes (table 1). Figure 2 summarises the interrelationship between these PTs, set in the wider context of structural and organisational barriers affecting autism diagnostic pathways. Full PTs with CMO configurations are provided as online supplemental document 3.

\section{PT1: listening and recognition}

Professionals had to balance early referral with parents' concerns so that they felt listened to and taken seriously $^{63132}$; parents were often the first to notice atypical patterns of development or behaviour in their child. ${ }^{631-34}$ Managing parental expectations ${ }^{35}$ and developing a co-operative relationship appeared to help manage this balance but 'was perceived to be particularly problematic because access to services is based on diagnosis, rather than an assessment of the child and family's needs ${ }^{35}$ (p215). From parents' perspective, one autism charity website suggested they 'develop a talent for making a polite nuisance of themselves (more properly known as 'advocacy')' to traverse barriers to referral ${ }^{34}$ (p29),

Additionally, greater autism awareness and training for frontline professionals, particularly general practitioners and teachers, alongside training in how, when and who to refer to ${ }^{6} 12323436-38$ was suggested as a strategy to improve early identification.

\section{PT2: referral and triaging}

Comprehensive information gathering preassessment reduced the number of contacts, assessment duration and total time taken to reach diagnosis. ${ }^{39}$ A systematic approach to information gathering ${ }^{12} 3840$ improved efficiency, but referrers also wanted feedback when referrals were declined. ${ }^{12} 3841$

Innovative approaches to triaging included: sufficient information gathering preassessment to enable same-day assessment in the context of tertiary services ${ }^{42-44}$; initial interview with an experienced clinician $^{45}$; community/neurodevelopmental paediatrician carrying out a General Developmental Assessment ${ }^{412}{ }^{46}$; assessment by CAMHS or a community paediatrician and SALT, then allocating to an abbreviated (local) or complex (specialist) pathway ${ }^{41}$; triage meetings across CAMHS and Child Development Services (CDS) ${ }^{41}$ However, whether these strategies constituted triaging or the first stage in the diagnostic pathway was arguable.

\section{PT3: diagnostic assessment}

Good practice in the UK $(\mathrm{NICE})^{12}$ recognises the importance of multidisciplinary assessment with use of information from parents, educational settings and direct observation/assessment of the child used as evidence alongside health professional assessment. However, services had different condition-specific remits, catchment areas and commissioning agreements. Where community paediatrics and mental health services were integrated and collocated in the same organisation this allowed a seamless transition, avoiding duplicated waits and enabling families to see all relevant professionals at once. $^{1842}$

Few papers clearly delineated the service pathway ${ }^{18} 35$ 40-42 4748 and within these were wide variations, including the balance of standardised assessments, observations and clinical judgement. As recommended by NICE, ${ }^{12}$ most services were multidisciplinary, and many offered a single point of access, bridging the autism-ADHD diagnostic divide. ${ }^{18} 42$ For example, Peterborough's integrated pathway provided assessments for ADHD and autism ${ }^{18} 42$ and combined a single point of access with a comprehensive skill mix, including access to therapies. This reduced the number of assessments per individual, saved time and money, and provided a better diagnostic experience. ${ }^{42}$ Another approach was to extend the role of available professions, for example, by training SALTs to carry out aspects of the assessment previously carried out by child psychiatrists. ${ }^{40}$ However, disadvantages of multidisciplinary assessment and/or multi-agency working included being labour intensive and $\operatorname{costly}^{13}$; being negatively affected by the dissonance between medical and educational paradigms ${ }^{47}$; and a 'perceived power differential' evidenced by the 'decisionmaking power of doctors and psychologists over other clinicians' ${ }^{49}$ (p322).

Rutherford $e t a t^{41}$ presented a multi-agency diagnostic pathway with an 'abbreviated' pathway when the signs and symptoms of autism were easily identified and a 'complex' pathway for CYP with, for example, coexisting conditions needing onward referral to a specialist team. This resulted in fewer CYP unnecessarily going through the full process, improving the timeliness of assessment. ${ }^{41}$

An interesting theme within the literature considered the balance of clinical expertise against standardised assessments. Less experienced clinicians appeared to prefer using standardised tools, while more experienced clinicians expressed confidence in their clinical judgement. ${ }^{45}$ Some clinicians found diagnostic tools helpful, while others described them as 'very cumbersome and very time consuming, ${ }^{47}$ (p118). Rogers et a $\tilde{D}^{0}$ referred to 'upgrading', whereby the majority of professionals (78 out of 116) erred on the side of a positive diagnosis when faced with uncertainty. The main reasons were to facilitate access to funding/support $(\mathrm{n}=17 ; 22 \%)$; enable individuals to get a statement of Special Educational Needs $(\mathrm{n}=8 ; 10 \%)$; or differing opinions among colleagues in a team $(\mathrm{n}=32 ; 41 \%)$.

Finally, there was limited but positive literature around the use of technology. Aims included 'remote' observational assessments carried out by families during a short telehealth assessment to screen for autism in children under 3 years ${ }^{51}$; using mobile technology to collect observational data in advance of formal assessment ${ }^{52}$; educational games to assess risk of autism ${ }^{52}$; an automated story 
Table 1 Programme theories and sources

PTs 1-4: Stage specific programme theories affecting the diagnostic assessment pathway

\section{PT1 Listening and recognition}

If frontline health and education professionals (eg, GPs, teachers) are confident in recognising the signs and symptoms of autism, are cognisant of referral pathways and listen to parents, taking their concerns seriously then CYP will be referred to an appropriate service, in a timely manner, reducing parental frustration.

\section{PT2 Referral and triaging}

If autism diagnostic services provide clear guidelines for referrers on what information is needed and how to refer, and referrers follow these guidelines, then time will be saved at the triaging stage and proportionately fewer CYP who do not have autism will go through the full process.

\section{PT3 Diagnostic assessment}

If a structured, consistent and multidisciplinary approach to service delivery is adopted, making best use of available staff and clinical expertise, then the number of assessments per individual may be reduced.

If a balance of interview, observation and recognised tools are used, alongside an assets-based approach, this will ensure a comprehensive and family-friendly diagnostic experience. If the same Trust manages both community paediatrics and mental health services, this potentially allows for a seamless transition, avoids duplicate waits and enables families to see all relevant professionals at the same time.
NICE, 2011 12; Reed and Osborne, 2012 ${ }^{78}$; Abbott et al, 2013 ${ }^{31}$; The Scottish Government, 2014 ${ }^{36}$; Crane et al, $2016^{6}$; Rogers et al, 2016 ${ }^{50}$; O'Reilly et al, 2017 ${ }^{32}$; RCPCH, 201720; Potter, 2017 ${ }^{33}$; Unigwe et al, 2017 ${ }^{37}$; Crane et al, 2018 ${ }^{58}$; Dowden, 2018 ${ }^{34}$; Rutherford et al, 2018 ${ }^{41}$; Ford et al, 201979; Hurt et al, 2019. ${ }^{35}$

NICE, 2011 ${ }^{12}$; Carpenter, 2012 ${ }^{45}$; The Scottish Government, 2014 ${ }^{36}$; McKenzie et al, 2015 ${ }^{39}$; Healthcare Improvement Scotland, 201657; Rutherford et al, 2016 ${ }^{38}$; Rutherford et al, 2018 ${ }^{41}$; Autistica, 2019 ${ }^{42}$; Hurt et al, 2019 ${ }^{35}$; Tollerfield and Pearce, 2020.40

Carpenter, 2012 ${ }^{45}$; NICE, 2014a ${ }^{60}$; Karim et al, 2014 ${ }^{47}$; Gray et al, 2015 ${ }^{48}$; Crane et al, 2016 ${ }^{6}$; Halpin, 2016 ${ }^{49}$; Healthcare Improvement Scotland, 2016 57 ; McKenzie et al, 2016 ${ }^{80}$; Rogers et al, 2016 ${ }^{50}$; Rutherford et al, 2016 ${ }^{38}$; Tryfona et al, 2016 ${ }^{52}$; Galliver et al, 2017 13 ; Jordan et al, 2017 $7^{53}$; Juárez et al, 2018 ${ }^{51}$; Rutherford et al, 2018 ${ }^{41}$; Ahlers et al, 2019 ${ }^{81}$; Autistica, 2019 ${ }^{42}$; Ford et al, 201979; Tollerfield and Pearce, 2020.40

\section{PT4 Diagnostic feedback}

If parents understand the diagnostic process and feel supported NICE, 2011 ${ }^{12}$; RASDN, 2011 ${ }^{82}$; Calzada et al, $2012^{62}$; this can moderate parental expectations. Feedback should take Carpenter, $2012^{45}$; Reed and Osborne, $2012^{78}$; Abbott et an assets-based approach and management plans should be individualised, taking account of co-existing conditions. Reports should be timely and in a format that everyone finds helpful. al, 2013 31 ; Karim et al, 2014 ${ }^{47}$; NICE, 2014a ${ }^{60}$; The Scottish Government, 2014 ${ }^{36}$; Halpin, 2016 ${ }^{49}$; Healthcare Improvement Scotland, 2016 ${ }^{57}$; Hennel et al, 2016 ${ }^{56}$; McKenzie et al, 2016 ${ }^{80}$; Reed et al, 2016 ${ }^{83}$; Rogers et al, 2016 ${ }^{50}$; Crane et al, 2018 ${ }^{58}$; The Scottish Government, $2018^{64}$; Autistica, 2019 ${ }^{42}$; Hurt et al, 2019 ${ }^{35}$; Tollerfield and Pearce, $2020 .^{40}$

\section{PTs 5-7: Cross-cutting programme theories affecting the diagnostic pathway}

\section{PT5: Working in partnership with families}

If parents have a single point of contact, are provided explanations throughout and included in decision-making then the diagnostic pathway may be less stressful.

\section{PT6: Interagency working}

If 'experts' including people with autism, carers, professionals and specialist organisations work in partnership and the knowledge generated is effectively embedded into local services, this will build capacity, improve parent/CYP satisfaction and support planning of services both locally and nationally.

\section{PT7: Training, service development and evaluation}

If professionals have access to tailored training based on their needs, competencies and role, and services engage in service development and evaluation, this will increase the local skill set of people who regularly work with CYP who may have autism.
Calzada et al, 2012 ${ }^{62}$; Abbott et al, 2013 ${ }^{31}$; Gregory et al, 2013b ${ }^{59}$; NICE, 2014a ${ }^{60}$; Rogers et al, 2016 ${ }^{50}$; Healthcare Improvement Scotland, $2016^{57}$; Crane et al, $2018^{58}$

NICE, 2011 ${ }^{12}$; Calzada et al, 201262; Gregory et al, 2013a ${ }^{61}$; Gregory et al, 2013b ${ }^{59}$; Karim et al, 2014 ${ }^{47}$; NICE, 2014a ${ }^{60}$; The Scottish Government, 2014 ${ }^{36}$; Gray et al, 2015 ${ }^{48}$; Healthcare Improvement Scotland, 2016 ${ }^{57}$; Rogers et al, 2016 ${ }^{50}$; Galliver et al, 2017 $7^{13}$; Hayes et al, 2018 ${ }^{84}$; The Scottish Government, $2018^{64}$; Williams et al, $2018^{71}$; Hurt et al, 201935; Tollerfield and Pearce, $2020 .^{40}$

NICE, 2011 ${ }^{12}$; Gregory et al, 2013a ${ }^{61}$; Autism ACHIEVE Alliance, 2014 ${ }^{63}$; NHS Education for Scotland, 2014 ${ }^{85}$; The Scottish Government, 2014 ${ }^{36}$; Rutherford et al, 2016 ${ }^{38}$; $\mathrm{RCPCH}, 2017^{20}$; Rutherford et al, 2018 ${ }^{41}$; The Scottish Government, 2018. ${ }^{64}$

CYP, children and young people; GPs, general practitioners. 


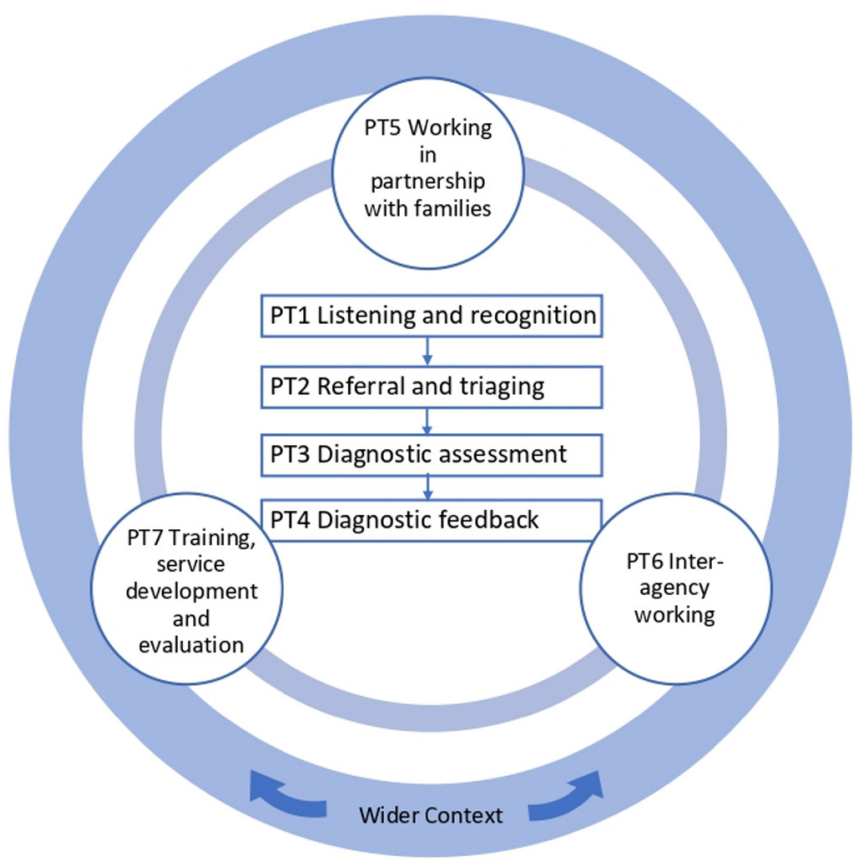

Figure 2 Programme theories (PTs) for the autism diagnostic pathway.

('A Pirates Adventure') scoring emotional cognition ${ }^{53}$; and the use of computer-based Continuous Performance Tests. ${ }^{54}$ Our expert stakeholders also suggested that where the presence of ADHD is suspected, the use of Qbtest $^{54}$ may enable an objective measurement of attention, concentration, impulsivity and distractibility but the evidence is limited. Since carrying out the RRR, Lord ${ }^{55}$ has provided guidance on adapting autism diagnostic assessment during social distancing, including the Autism Diagnostic Observation Schedule (although unvalidated), for remote use, demonstrating that the current COVID-19 crisis has become a driver for telehealth approaches.

\section{PT4: diagnostic feedback}

Most parents regarded autism diagnosis as a gateway to services ${ }^{50}$ but there was no consensus on best practice regarding feedback. ${ }^{48}$ Parents valued a sensitive approach and positive comments about their child and their parenting $^{31}$ but found it hard to absorb feedback. ${ }^{31} 56$ Practical strategies included a structured approach; using consistent and straightforward terminology; opportunity to ask questions (including later); and recognising their child's skills/strengths. ${ }^{12} 31475657$ Guidelines recommended a needs-based and tailored management plan, co-developed with parents. ${ }^{12}$

Only one paper provided detailed information on the report format ${ }^{40}$ and used a digital report-writing tool and visual profiling tool. Reports were available within a few days, enabling parents to review the content, improving partnership working. The visual profiling tool provided a concise visual aide for understanding, explaining, and communicating the abilities of each CYP.

\section{PT5: working in partnership with families}

The diagnostic process was enhanced by integrating 'expertise from several perspectives... that of the individual, their family, and the professionals ${ }^{58}$ (p3762) and acknowledging parents as co-experts. When parents understood the diagnostic process in advance, this improved satisfaction and helped moderate expectations. ${ }^{31}$ Open and honest dialogue involving parents in decision-making, ${ }^{50}$ helped promote engagement and manage differences of opinion. ${ }^{59}$ Having a named 'case coordinator' ${ }^{12}$ or 'keyworker ${ }^{60}$ helped reduce stress and increase engagement. ${ }^{59}$ Parents offered support following diagnosis were, unsurprisingly, more satisfied than those who were not. ${ }^{58} \mathrm{~A}$ simple suggestion to improve satisfaction was to tailor links to relevant services and explore the full range of services that might prove useful. ${ }^{6}$ Another approach was to help parents develop strategies to manage difficulties, for example, meeting families wherever most convenient to reduce non-attendance. ${ }^{59}$

\section{PT6: interagency working}

Integrating the pathways into a single assessment process potentially saved time and cost less ${ }^{1318}{ }^{21}$ but we found little evidence of how to address macro-level constraints such as chronic underinvestment. ${ }^{34}$ Much appeared to rest on personal relationships at the micro-level ${ }^{61}$ and/ or parents co-ordinating services. ${ }^{35}$ While joint working was endorsed ${ }^{62}$ suggestions to promote it were limited to establishing clear pathways ${ }^{63}$; creating opportunities to work in different teams, such as split posts or secondments $^{59}$; and an Additional Learning Needs Coordinator (a teacher at the school). ${ }^{35}$

\section{PT7: training, service evaluation and development}

Several papers identified the importance of training in improving the quality and efficiency of autism diagnostic services. ${ }^{36}{ }^{41}$ It was recommended that training should go beyond those working in autism services, include the educational sector ${ }^{64}$ and be geared to the needs of managers as well as frontline staff ${ }^{36}$ through multi-agency training. ${ }^{12}$

Rutherford $e t a t^{11}$ advocated a training framework with different skill levels, depending on the "nature, extent and likely impact of daily contact with individuals with ASD $^{41}$ (p1583) and now reflected in Health Education England recommendations. ${ }^{65}$ Other training suggestions included an opportunity to observe specialist autism services; buddying with experienced clinicians; regular review of training needs and succession planning; and a national forum to share experiences and knowledge. ${ }^{38} 63$

Finally, service evaluation was advocated to check adherence to standards/guidelines ${ }^{20}$ and provide evidence for commissioners ${ }^{38}$; one strategy was a guidelines checklist at the front of each patient file. ${ }^{38}$ Service development suggestions included having one person to champion change; generating research within clinical teams; encouraging practitioners to co-create contextually sensitive solutions ${ }^{38}$; and drawing on the expertise 
of people with autism, carers and specialist organisations. ${ }^{36}$ Our stakeholders highlighted the importance of good quality national data to facilitate a whole system approach, with the current approach appearing somewhat fragmented. ${ }^{66}$

\section{DISCUSSION}

This RRR explored diagnostic pathways that have been adopted across the UK, to determine what works best, for whom and in what circumstances. Four PTs related to the clinical pathway, addressing ways to improve initial recognition of possible autism, referral and triaging, the diagnostic model and post-diagnostic feedback. While there were specific service delivery innovations of interest, such as adopting a broader neurodevelopmental approach to assessment, or the use of skill mix, there also appears to be scope to adapt stages within the process. For example, gathering information about a CYP's strengths/needs at the point of referral may enhance the process, regardless of the specific model. The three cross-cutting PTs centred on working in partnership with families; interagency working; and training, service evaluation and development. Collectively, these PTs evidence different approaches that could contribute to a better experience for families, improved efficiency (and potentially cost savings) and shorter waiting lists.

Many of the issues identified in the RRR could be addressed by full adherence to NICE guidelines ${ }^{12}$ and quality standards. ${ }^{67}$ However, a gap exists between guidelines and local interpretation, exacerbated by demand for assessment outstripping capacity and resourcing constraints. In particular, the guidelines indicate the need for a team with the competencies to deliver a broader neurodevelopmental and mental health assessment, producing a comprehensive description of a chid's strengths and needs, but some services appeared focused solely on autism diagnosis, partly reflecting resourcing constraints. ${ }^{36}$ A broader neurodevelopmental approach ${ }^{58}$ may also ameliorate the concerns of those families whose child does not meet criteria for an autism diagnosis but has significant needs which may otherwise remain, or feel, unrecognised. This would be additionally aided by clinical teams resourcing the development of strengths and needs planning or working in consort with other agencies.

As previously noted, there may also be a trade-off between carrying out comprehensive assessments for all CYP with possible autism and 'providing a more streamlined approach that is tailored to the child's presentation'$^{\prime 68}$ (p526) which could reduce diagnostic validity. This mirrors feedback from our expert stakeholdersthat there may need to be a discussion around the potential to increase investment in service delivery to enable high quality and timely approach versus the potential challenges associated with accepting lower quality and less timely diagnostic assessment. A similar approach delivering tiered assessment according to diagnostic complexity, has been recommended by recent Australian guidelines. $^{69}$

While the study findings are based on UK literature that relates to the NHS where health provision is free at the point of care, and insurance-based health economies are different, ${ }^{68}$ the international literature was largely consistent with our findings. For example, recommendations to engage families in service design, and to produce a needs-based holistic assessment and report are mirrored internationally. ${ }^{69} 70$ The seven PTs are echoed overall, for example in New Zealand recommendations, ${ }^{71}$ while international research also supports individual PTs, including improving knowledge and skills of referrers,${ }^{72}$ improving information gathering to inform appropriateness of referral, ${ }^{54}$ and upskilling the diagnostic workforce. ${ }^{73} 74$ These are also echoed in recommendations from NHS England published after completion of the RRR. ${ }^{75}$

Internationally, digitally delivered training programmes such as Extension for Community Healthcare Outcomes have been developed to enable upskilling of a wider diagnostic workforce, for example community general paediatricians in USA and Canada ${ }^{74}$ while the WHO has developed Caregiver Skills Training Programmes to train parents to support their children's development. ${ }^{76}$ Similarly, the need for social distancing during the COVID-19 pandemic has acted as a driver to adopt digital technologies, although some of these had already been developed in response to geographical distancing between centralised specialist services and families living in widespread rural communities. ${ }^{57}$

\section{Implication for practice and future research}

From the PTs we identified six key areas that would benefit from further exploration. These were evaluation of: training and support materials available for non-specialist staff and parents/CYP accessing the diagnostic pathway which would increase early recognition that a child may need assessment and improve information gathering at the point of referral; training packages to upskill those working in autism services and the subsequent impact on workforce shortages; asset-based approaches to diagnosis, management and support; barriers and facilitators to comprehensive needs-led diagnostic assessment; approaches to integrating services dealing with autism; and increased use of technology in assessment that has already started in the context of COVID-19. ${ }^{77}$

\section{Strengths and limitations}

The realist approach was well suited to examining and understanding the complexity of autism diagnostic assessment, and the challenges of delivering such services in different contexts. We developed systematic and focused search strategies, within the parameters of $\mathrm{RRR},{ }^{22}$ although not as extensive as a full realist review. Expert Stakeholder Engagement enhanced the search strategy, enabled an iterative approach to identifying relevant literature and was invaluable when synthesising 
the findings. Most papers had limited information on care pathway processes and contextual factors (which in realist terminology refers to any trigger that influences responses or resources), or more general subanalysis by demographic/other characteristics, so PTs could only develop based on what was reported; this highlights the need for further empirical work which the next phase of this study will provide. Primary and background searches were restricted to UK only, given UK NHS context, but secondary searches included papers from countries with somewhat similar healthcare systems (USA, Canada, Australia, New Zealand) to help elucidate findings, as recommended by our expert stakeholders. However, we acknowledge that we may have missed literature from similar health systems that could have informed our PTs.

\section{CONCLUSION}

In conclusion, this RRR identified important aspects that may contribute to more efficient, high quality and familyfriendly service delivery. We will test the PTs and how service design could be further enhanced in the subsequent stages of the wider RE-ASCeD study.

\section{Author affiliations}

${ }^{1}$ Centre for Health Service Studies, University of Kent, Canterbury, UK

${ }^{2}$ Sussex Community NHS Foundation Trust, Brighton, UK

${ }^{3}$ Brighton and Sussex Medical School, Brighton, UK

${ }^{4}$ Peterborough Child Development Centre, Cambridgeshire and Peterborough NHS

Foundation Trust, Fulbourn, UK

${ }^{5}$ Newcastle University Population Health Sciences Institute, Newcastle upon Tyne, UK

${ }^{6}$ Cumbria, Northumberland Tyne and Wear NHS Foundation Trust, Newcastle upon Tyne, UK

${ }^{7}$ Newcastle upon Tyne Hospitals NHS Foundation Trust, Newcastle upon Tyne, UK

Twitter Vanessa Abrahamson vabrahamson@UoK and Wenjing Zhang @ Wenjingzhang_

Acknowledgements We would like to thank other members of the Realist Evaluation of Autism ServiCe Delivery (RE-ASCeD) Consortium for their consultation and support: Amanda Allard, Assistant Director, Council for Disabled Children; Prof Heather Gage, University of Surrey; Dr Victoria Grahame, Cumbria, Northumberland Tyne and Wear NHS Trust; Dr Lorcan Kenny, Autistica; Dr Gráinne Saunders, West Sussex Parent Carer Forum; Peter Williams, University of Surrey; Kat Wilmore, PPI partner; and members of our Expert Advisory Group.

Contributors VA/WZ: involved in all stages of the review and writing all drafts of this paper. VA acting as guarantor. PW: substantial contribution to writing protocol for the overall RE-ASCeD project, all stages of the review and commenting on all drafts of this paper. WF/IM: substantial contribution to writing protocol for the overall RE-ASCeD project, all stages of the review and commenting on drafts of this paper. JP: substantial contribution to writing protocol for the overall RE-ASCeD project, some stages of the review and commenting on a draft of this paper. VR: substantial contribution to writing protocol for the overall RE-ASCeD project, some stages of the review and commenting on a draft of this paper. AP: designing the search strategy and commenting on the methodology section of this paper. VA acts as a gaurantor.

Funding This work was supported by NHS England and funds were derived from the child and young person mental health transformation funding stream, via the Learning Disability and Autism Directorate (direct quote from NHS England letter dated 28/8/2019). There is no grant number.

Competing interests None declared.

Patient consent for publication Not applicable.
Provenance and peer review Not commissioned; externally peer reviewed.

Data availability statement Data are available upon reasonable request. All data relevant to the study are included in the article or uploaded as supplementary information.

Supplemental material This content has been supplied by the author(s). It has not been vetted by BMJ Publishing Group Limited (BMJ) and may not have been peer-reviewed. Any opinions or recommendations discussed are solely those of the author(s) and are not endorsed by BMJ. BMJ disclaims all liability and responsibility arising from any reliance placed on the content. Where the content includes any translated material, BMJ does not warrant the accuracy and reliability of the translations (including but not limited to local regulations, clinical guidelines, terminology, drug names and drug dosages), and is not responsible for any error and/or omissions arising from translation and adaptation or otherwise.

Open access This is an open access article distributed in accordance with the Creative Commons Attribution Non Commercial (CC BY-NC 4.0) license, which permits others to distribute, remix, adapt, build upon this work non-commercially, and license their derivative works on different terms, provided the original work is properly cited, appropriate credit is given, any changes made indicated, and the use is non-commercial. See: http://creativecommons.org/licenses/by-nc/4.0/.

\section{ORCID iDs}

Vanessa Abrahamson http://orcid.org/0000-0002-1169-9457

Wenjing Zhang http://orcid.org/0000-0002-1810-791X

Patricia M Wilson http://orcid.org/0000-0002-5787-9736

William Farr http://orcid.org/0000-0003-3644-5311

Venkat Reddy http://orcid.org/0000-0002-3512-0171

Jeremy Parr http://orcid.org/0000-0002-2507-7878

Anna Peckham http://orcid.org/0000-0003-2342-5442

lan Male http://orcid.org/0000-0001-5426-6646

\section{REFERENCES}

1 Rodgers H, McCluney J. The prevalence of autism (including Asperger syndrome) in school age children in Northern Ireland 2021. Belfast, Northern Ireland: Information \& Analysis Directorate, Department of Health, 2021.

2 Roman-Urrestarazu A, van Kessel R, Allison C, et al. Association of race/ethnicity and social disadvantage with autism prevalence in 7 million school children in England. JAMA Pediatr 2021;175:e210054-e.

3 Maenner MJ, Shaw KA, Baio J, et al. Prevalence of autism spectrum disorder among children aged 8 years - autism and developmental disabilities monitoring network, 11 sites, United States, 2016. MMWR Surveill. Summ. 2020;69:1-12.

4 Brett D, Warnell F, McConachie $\mathrm{H}$, et al. Factors affecting age at ASD diagnosis in UK: no evidence that diagnosis age has decreased between 2004 and 2014. J Autism Dev Disord 2016;46:1974-84.

5 Wigham S, Male I, Farr W. UK childhood autism diagnostic services survey 2020: challenges and innovations. Archives of Disease in Childhood. In Press 2021.

6 Crane L, Chester JW, Goddard L, et al. Experiences of autism diagnosis: a survey of over 1000 parents in the United Kingdom. Autism 2016;20:153-62.

7 NHS England. NHS long term plan: learning disability and autism. section 3.31-3.36, 2019. Available: https://www.longtermplan.nhs.uk/ areas-of-work/learning-disability-autism/

8 American Psychiatric Association. Diagnostic and statistical manual of mental disorders. Fifth ed. Arlington, VA: American Psychiatric Association, 2013.

9 Simonoff E, Pickles A, Charman T, et al. Psychiatric disorders in children with autism spectrum disorders: prevalence, comorbidity, and associated factors in a population-derived sample. J Am Acad Child Adolesc Psychiatry 2008;47:921-9.

10 Joshi G, Petty C, Wozniak J, et al. The heavy burden of psychiatric comorbidity in youth with autism spectrum disorders: a large comparative study of a psychiatrically referred population. J Autism Dev Disord 2010;40:1361-70.

11 Wistow R, Barnes D. A profile of child and adolescent mental health services in England 2007/8, 2009. Available: https://dro.dur.ac.uk/ 8221/1/8221.pdf

12 National Institute for Health and Care Excellence. Autism spectrum disorder in under 19s: recognition, referral and diagnosis (CG128) London, UK, 2011: 1-45. https://www.nice.org.uk/guidance/cg128

13 Galliver M, Gowling E, Farr W, et al. Cost of assessing a child for possible autism spectrum disorder? An observational study 
of current practice in child development centres in the UK. BMJ Paediatr Open 2017;1:e000052.

14 Male I, Farr W, Gain A. How much does it cost to assess a child for possible autism spectrum disorder in the UK National health service: an observational study. European Academy of Childhood Disability, 2019.

15 Vanhaecht K, Sermeus W, Van Zelm R. Care pathways are defined as complex interventions. BMC Medicine 2010;8:31.

16 Palmer E, Ketteridge C, Parr JR, et al. Autism spectrum disorder diagnostic assessments: improvements since publication of the National autism plan for children. Arch Dis Child 2011;96:473-5.

17 Male I, Reddy V. Should ADHD, ASD \& related services be delivered in an integrated way? BACCHNEWS, 2018.

18 Male I, Farr W, Reddy V. Autism or related conditions be delivered in an integrated neurodevelopmental pathway? J Integr HealthCare 2020;2:e000037.

19 Abrahamson V, Zhang W, Wilson P, et al. Realist evaluation of autism service delivery (RE-ASCeD): which diagnostic pathways work best, for whom and in what context? protocol for a rapid realist review. BMJ Open 2020;10:e037846.

$20 \mathrm{RCPCH}$. Invited reviews the first four years, 2017. Available: https:// www.rcpch.ac.uk/sites/default/files/Invited_Reviews_evaluation report_2016.pdf

$21 \mathrm{BACCH}$. A workforce strategy for community paediatrics. London: British association for community child health, 2019.

22 Saul JE, Willis CD, Bitz J, et al. A time-responsive tool for informing policy making: rapid realist review. Implement Sci 2013;8:103.

23 NIHR Centre for Engagement and Dissemination. Uk standards for public involvement, 2021. Available: https://sites.google.com/nihr.ac. uk/pi-standards/home

24 Wong G, Greenhalgh T, Westhorp G, et al. RAMESES publication standards: realist syntheses. BMC Med 2013;11:21

25 Williams AC. Autoextraction of twelve permanent teeth in a child with autistic spectrum disorder. Int J Paediatr Dent 2016;26:157-9.

26 Weetman K, Wong G, Scott E, et al. Improving best practise for patients receiving hospital discharge letters: a realist review protocol. BMJ Open 2017;7:e018353.

27 Gilmore B, McAuliffe E, Power J, et al. Data analysis and synthesis within a realist evaluation: toward more transparent methodological approaches. Int J Qual Methods 2019;18:1609406919859754

28 Pawson R. Digging for Nuggets: How 'Bad' Research Can Yield 'Good' Evidence. Int J Soc Res Methodol 2006;9:127-42.

29 Pearson M, Brand SL, Quinn C, et al. Using realist review to inform intervention development: methodological illustration and conceptua platform for collaborative care in offender mental health. Implement Sci 2015;10:134

30 Willis CD, Saul JE, Bitz J, et al. Improving organizational capacity to address health literacy in public health: a rapid realist review. Public Health 2014:128:515-24.

31 Abbott M, Bernard P, Forge J. Communicating a diagnosis of Autism Spectrum Disorder - a qualitative study of parents' experiences. Clin Child Psychol Psychiatry 2013;18:370-82.

32 O'Reilly M, Nina Lester J, Muskett T, et al. How parents build a case for autism spectrum disorder during initial assessments: 'We're fighting a losing battle'. Discourse Stud 2017;19:69-83.

33 Potter CA. "I received a leaflet and that is all": Father experiences of a diagnosis of autism. Br J Learn Disabil 2017;45:95-105.

34 Dowden A. Improving the diagnosis of autism spectrum disorder. Prescriber, 2018: 28-30.

35 Hurt L, Langley K, North K, et al. Understanding and improving the care pathway for children with autism. Int J Health Care Qual Assur 2019;32:208-23.

36 The Scottish Government. Scottish strategy mapping report, 2014. Available: http://www.autismstrategyscotland.org.uk/strategy/keydocuments.html

37 Unigwe S, Buckley C, Crane L, et al. GPs' confidence in caring for their patients on the autism spectrum: an online self-report study. $\mathrm{Br}$ $J$ Gen Pract 2017;67:e445-52.

38 Rutherford M, McKenzie K, Forsyth K, et al. Why are they waiting? Exploring professional perspectives and developing solutions to delayed diagnosis of autism spectrum disorder in adults and children. Res Autism Spectr Disord 2016;31:53-65.

39 McKenzie K, Forsyth K, O'Hare A, et al. Factors influencing waiting times for diagnosis of autism spectrum disorder in children and adults. Res Dev Disabil 2015;45-46:300-6.

40 Tollerfield I, Pearce H. Use of the thinking patterns in autism profiling model within a diagnostic assessment service for autism. Good Autism Practice 2020;21:99-117.

41 Rutherford M, Burns M, Gray D, et al. Improving efficiency and quality of the children's ASD diagnostic pathway: lessons learned from practice. J Autism Dev Disord 2018;48:1579-95.
42 Autistica. Embracing complexity in diagnosis: multi diagnostic pathways for NDCs, 2019. Available: https://embracingcomplexity. org.uk/

43 GOSH Foundation Trust. Neurodevelopmental assessment clinic (including the autism assessment service. Contract No.: Ref: 2018F0719. London, 2018.

44 Cumbria Northumberland Tyne and Wear NHS Foundation Trust. Complex neurodevelopmental disorders service (CNDS) for children and young people, 2018. Available: https://www.cntw.nhs.uk/ resource-library/complex-neuro-developmental-service-cnds/

45 Carpenter P. Diagnosis and assessment in autism spectrum disorders. Adv Ment Health Intellect Disabil 2012;6:121-9.

46 Male I, Farr W, Gowling E. How much does it cost the NHS to assess a child for possible autism: a prospective study - Paul Polani Award Lecture. British Academy of Childhood Disability Annual Conference, 2018.

47 Karim K, Cook L, O'Reilly M. Diagnosing autistic spectrum disorder in the age of austerity. Child Care Health Dev 2014;40:115-23.

48 Gray L, Gibbs J, Jolleff N, et al. Variable implementation of good practice recommendations for the assessment and management of UK children with neurodisability. Child Care Health Dev 2015;41:938-46.

49 Halpin J. What do nurses think they are doing in pre-school autism assessment? Br J Nurs 2016;25:319-23.

50 Rogers CL, Goddard L, Hill EL, et al. Experiences of diagnosing autism spectrum disorder: a survey of professionals in the United Kingdom. Autism 2016;20:820-31.

51 Juárez AP, Weitlauf AS, Nicholson A, et al. Early identification of ASD through telemedicine: potential value for underserved populations. $J$ Autism Dev Disord 2018;48:2601-10.

52 Tryfona C, Oatley G, Calderon AAntona M, Stephanidis C, eds. $M$-Health solutions to support the National health service in the diagnosis and monitoring of autism spectrum disorders in young children. Universal Access in Human-Computer Interaction: Users and Context Diversity, Pt lii. Lecture Notes in Computer Science, 2016: 249-56.

53 Jordan E, Farr W, Fager S. Pirate adventure autism assessment app: a new tool to aid clinical assessment of children with possible autistic spectrum disorder. In: Powell W, Rizzo A, Sharkey P, eds. Rehabilitation: innovation and challenges in the use of virtual reality technologies. NY, US: Nova Publishers, 2017: 27-38.

54 QbTech. ADHD tests, 2020. Available: https://www.qbtech.com/ adhd-tests\#

55 Lord C. Assessments in this time of social distancing, 2020. Available: https://www.youtube.com/watch?v=sOGv8vbJeeo

56 Hennel S, Coates C, Symeonides C, et al. Diagnosing autism: Contemporaneous surveys of parent needs and paediatric practice. $J$ Paediatr Child Health 2016;52:506-11.

57 Healthcare Improvement Scotland. Sign 145: assessment, diagnosis and interventions for autism spectrum disorders [1-83], 2016. Available: https://www.sign.ac.uk/sign-145-assessment,-diagnosisand-interventions-for-autism-spectrum-disorders.html

58 Crane L, Batty R, Adeyinka H, et al. Autism diagnosis in the United Kingdom: perspectives of autistic adults, parents and professionals. J Autism Dev Disord 2018:48:3761-72.

59 Gregory S, Arnold T, Sharman C. The development of a child and adolescent mental health service for children with disabilities: rationale for the approach, method and techniques. Good Autism Practice 2013b;14:74-9.

60 National Institute for Health and Care Excellence. CG128 Surveillance report - recommendation for guidance executive 2014a [1-98]. Available: https://www.nice.org.uk/guidance/cg128/update/ CG128/documents/cg128-autism-in-children-and-young-peoplesurveillance-review-decision2

61 Gregory S, Arnold T, Sharman C. The development of a child and adolescent mental health service specifically for children with disabilities: reflections on the first four years. Good Autism Practice 2013a:14:68-73.

62 Calzada LR, Pistrang N, Mandy WP, et al. and Asperger's disorder: Utility and meaning for families. Journal of Autism and Developmental Disorders 2012;42:230-43.

63 Autism ACHIEVE Alliance. ASD: Waiting for Assessment - Executive Summary, 2014. Available: http://www.autismstrategyscotland.org. uk/strategy/key-documents.html

64 The Scottish Government. The Scottish Strategy for Autism Engagement Analysis 2018 Edinburgh, 2018. Available: http://www. autismstrategyscotland.org.uk/strategy/key-documents.html

65 Health Education England. Advanced clinical practice: capabilities framework when working with people who have a learning disability and/or autism, 2020. Available: https://skillsforhealth.org.uk/info-hub/ learning-disability-and-autism-frameworks-2019/ 
66 Allard A, Fellowes A, Gardiner A, et al. It takes leaders to break down siloes. integrating services for disabled children, 2019. Available: https://councilfordisabledchildren.org.uk/help-resources/resources/ it-takes-leaders-break-down-siloes-cdcs-new-report-integratingservices

67 National Institute for Health and Care Excellence. Qs 51 autism quality standard 2014b. Available: https://www.nice.org.uk/guidance/ qs51

68 Penner M, Anagnostou E, Andoni LY, et al. Systematic review of clinical guidance documents for autism spectrum disorder diagnostic assessment in select regions. Autism 2018;22:10.1177/1362361316685879:517-27.

69 Whitehouse A, Evans K, Eapen V, et al. A national guideline for the assessment and diagnosis of autism spectrum disorders in Australia, 2018. Available: https://www.autismcrc.com.au/knowledge-centre/ resource/national-guideline

70 van der Meer L, Evans K. The autism diagnostic process in New Zealand: final report. Brisbane Autism CRC, 2021.

71 Williams AN, Mold B, Kilbey L, et al. Forty years of referrals and outcomes to a UK child development centre (CDC): has demand plateaued? Child Care Health Dev 2018;44:10.1111/ cch.12552:364-9.

72 Gordon-Lipkin E, Foster J, Peacock G. Whittling down the wait time: exploring models to minimize the delay from initial concern to diagnosis and treatment of autism spectrum disorder. Pediatr Clin North Am 2016;63:851-9.

73 Swanson AR, Warren ZE, Stone WL, et al. The diagnosis of autism in community pediatric settings: does advanced training facilitate practice change? Autism 2014;18:555-61.

74 Sohl K, Mazurek MO, Brown R. Echo autism: using technology and mentorship to bridge gaps, increase access to care, and bring best practice autism care to primary care. Clin Pediatr 2017;56:509-11.

75 NHSE and NHSI autism program strategy group. Autism diagnosis and support deep dives. NHS England and NHS Improvement, 2020.
76 Salomone E, Pacione L, Shire S, et al. Development of the who caregiver skills training program for developmental disorders or delays. Front Psychiatry 2019;10:769

77 Reddy V, Brumpton L. Digital-driven service improvement during the COVID-19 pandemic. Paediatrics and Child Health, 2021: 1-3.

78 Reed P, Osborne LA. Diagnostic practice and its impacts on parental health and child behaviour problems in autism spectrum disorders. Arch Dis Child 2012;97:927-31.

79 Ford T, Kenchington R, Norman S, et al. The agreement between the referrer, practitioner and research diagnosis of autistic spectrum conditions among children attending child and adolescent mental health services. Eur Child Adolesc Psychiatry 2019;28:1253-64.

80 McKenzie K, Forsyth K, O'Hare A, et al. The relationship between waiting times and 'adherence' to the Scottish Intercollegiate Guidelines Network 98 guideline in autism spectrum disorder diagnostic services in Scotland. Autism 2016;20:395-401.

81 Ahlers K, Gabrielsen TP, Ellzey A, et al. A pilot project using pediatricians as initial diagnosticians in multidisciplinary autism evaluations for young children. J Dev Behav Pediatr 2019;40:1-11.

82 RASDN. Six Steps of Autism Care - for Children and Young People in Northern Ireland, 2011. Available: https://www.familysupportni. gov.uk/Content/uploads/userUploads/ASD\%20-\%20Six\%20Steps\% 20of\%20Autism\%20Care\%20Pathway\%20Report\%20-\%20PDF\% 203MB.pdf

83 Reed P, Picton L, Grainger N, et al. Impact of diagnostic practices on the self-reported health of mothers of recently diagnosed children with ASD. Int J Environ Res Public Health 2016;13:07.

84 Hayes J, Ford T, Rafeeque $\mathrm{H}$, et al. Clinical practice guidelines for diagnosis of autism spectrum disorder in adults and children in the UK: a narrative review. BMC Psychiatry 2018;18:1-25.

85 NHS Education for Scotland. The NHS Education for Scotland Autism Training Framework - Optimising Outcomes - A framework for all staff working with people with Autism Spectrum Disorders, their families and carers. Edinburgh, 2014. Available: https://www. knowledge.scot.nhs.uk/media/12392691/sct0117216030-3\% 20 asd \%20training\%20framework\%20cov final.pdf 